\title{
Recent developments in neutron imaging with applications for porous media research
}

\author{
Anders P. Kaestner ${ }^{1}$, Pavel Trtik ${ }^{1}$, Mohsen Zarebanadkouki ${ }^{2}$, Daniil Kazantsev ${ }^{3,4}$, Michal Snehota ${ }^{5}$, \\ Katherine J. Dobson ${ }^{6,7}$, and Eberhard H. Lehmann ${ }^{1}$ \\ ${ }^{1}$ Laboratory for Neutron Scattering and Imaging, Paul Scherrer Institut, Villigen, Switzerland \\ ${ }^{2}$ Division of Soil Hydrology, University of Goettingen, Goettingen, Germany \\ ${ }^{3}$ Manchester X-Ray Imaging Facility, School of Materials, University of Manchester, Manchester, UK \\ ${ }^{4}$ Manchester X-Ray Imaging Facility, Research Complex at Harwell, Didcot, UK \\ ${ }^{5}$ Faculty of Civil Engineering, Czech Technical University in Prague, Prague, Czech Republic \\ ${ }^{6}$ Ludwig-Maximilians Universität München, Department für Geo-und Umweltwissenschaften, 80333 Munich, Germany \\ ${ }^{7}$ Department of Earth Sciences, Durham University, Durham, DH1 3LE, UK
}

Correspondence to: Anders P. Kaestner (anders.kaestner@psi.ch)

Received: 30 October 2015 - Published in Solid Earth Discuss.: 4 December 2015

Revised: 3 June 2016 - Accepted: 11 July 2016 - Published: 6 September 2016

\begin{abstract}
Computed tomography has become a routine method for probing processes in porous media, and the use of neutron imaging is especially suited to the study of the dynamics of hydrogenous fluids, and of fluids in a highdensity matrix. In this paper we give an overview of recent developments in both instrumentation and methodology at the neutron imaging facilities NEUTRA and ICON at the Paul Scherrer Institut. Increased acquisition rates coupled to new reconstruction techniques improve the information output for fewer projection data, which leads to higher volume acquisition rates. Together, these developments yield significantly higher spatial and temporal resolutions, making it possible to capture finer details in the spatial distribution of the fluid, and to increase the acquisition rate of 3-D CT volumes. The ability to add a second imaging modality, e.g., X-ray tomography, further enhances the feature and process information that can be collected, and these features are ideal for dynamic experiments of fluid distribution in porous media. We demonstrate the performance for a selection of experiments carried out at our neutron imaging instruments.
\end{abstract}

\section{Introduction}

The knowledge of how hydrous fluids are redistributed within a pore network is central to many porous media experiments, and 3-D imaging using a variety of different imaging modalities (e.g., X-ray and neutron tomography) has become a useful tool since it can provide information about the displacements of fluids as well as the solid structures of the pore network within the sample. X-rays (XCT) are currently the most widely used imaging modality because of the high spatial and temporal resolution that can be achieved at modern synchrotron X-ray sources (e.g., Wildenschild and Sheppard, 2013; Cnudde and Boone, 2013; Dobson et al., 2016) and the relative availability of laboratory X-ray CT systems. However, neutron imaging (Anderson et al., 2009) has also proven very useful for in situ studies (e.g., Kardjilov et al., 2011). Neutron imaging is generally less accessible as there are fewer neutron imaging facilities worldwide (Lehmann and Kaestner, 2009), and while the lower fluxes mean it cannot always compete with X-ray imaging in speed, it has a distinct advantage in the excellent fluid-matrix contrast that arises from the high sensitivity to hydrogen. This difference comes from the neutron matter interaction, which is sensitive to the nucleus constellation instead of the electron shells as with X-rays (Krane, 1988). The protons and neutrons are also organized in shells and the cross section 
depends on the degree of completion of these shells. This makes neutrons an isotope-sensitive probe. Neutron imaging (both radiography and tomography) can quantify very small amounts of fluid, even when the pore space itself cannot be resolved, as demonstrated in several porous media studies (Schaap et al., 2008; Carminati et al., 2007; Robinson et al., 2008; Rudolph et al., 2012; Zarebanadkouki et al., 2014; Zhang et al., 2010; Lal et al., 2014). It is particularly relevant for investigations of water displacement in bulk samples where the detailed structure of the pore space is known, is unchanging, or is of lesser importance in understanding the fluid processes. The isotope sensitivity gives almost an order of magnitude in contrast between hydrogen $\left({ }^{1} \mathrm{H}\right)$ and deuterium $\left({ }_{2} \mathrm{H}\right.$ or $\left.\mathrm{D}\right)$. This allows isotopic tracing of an individual pulse of water through a preconditioned porous medium, identifying the spatial and temporal evolution of mixing behavior of fluids of similar density and viscosity (Zarebanadkouki et al., 2012). Furthermore, most metals are not opaque to neutrons (in contrast to X-rays), and are less opaque than the fluids. This means it is possible to observe fluid flow in situ within metal jacketed pressure and/or high-temperature vessels.

The flexibility of the instrumentation at neutron imaging beamlines meets the needs of a wide user community, and can cater for both large and small samples with a tradeoff between sample size and (spatial and temporal) resolution. Ongoing instrument development is steadily pushing the frontiers in capability and making new experiments possible. Here, we review recent advances in spatial and temporal performance at NEUTRA (Lehmann et al., 2001), ICON (Kaestner et al., 2011a), and BOA (Morgano et al., 2014) neutron imaging instruments at the Swiss spallation Neutron Source (SINQ), Paul Scherrer Institut (PSI). We provide a suite of examples to demonstrate state-of-the-art neutron imaging methods and instrument features that can be provided on demand through the ordinary user program, with a particular emphasis on those ideal for geological and porous media experiments.

\section{Methods and applications}

The individual radiographs collected during neutron imaging represent the neutrons that were transmitted through the sample. The mechanism that prevents the neutrons from reaching the detector is a combination of absorption and scattering. Scattering is often the dominant component of the neutron attenuation coefficient (Sears, 1992), and some neutrons reach the detector after multiple scattering events within the sample and instrument environment. The contribution of scattered neutrons must be considered if the experiment objective includes quantifying sample structure and fluid distribution with high accuracy (Hassanein, 2006). The images are mainly collected on a scintillator-based system with a cooled precision camera carrying either a CCD (charge-coupled de-

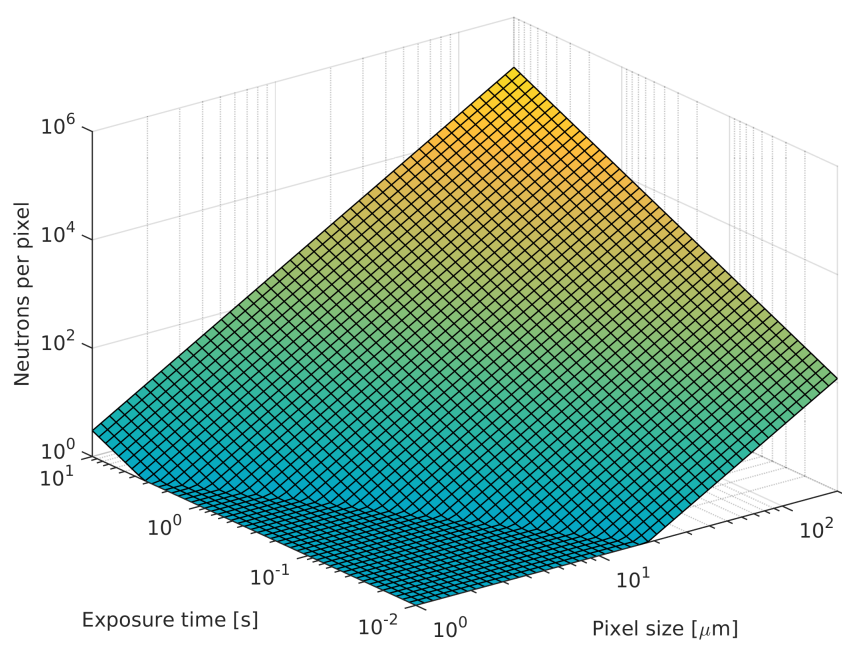

Figure 1. Average number of neutrons per pixel as a function of exposure time and pixel size calculated for a neutron flux of $10^{7}$ neutrons $\mathrm{cm}^{-2} \mathrm{~s}^{-1}$.

vice) or a sCMOS (scientific complementary metal-oxide semiconductor) detector chip. The two chip technologies have different advantages and complement each other. CCD chips have higher quantum efficiency and larger full well depth, making them ideal for long time exposures. In contrast, the sCMOS has a much shorter readout time, allowing high frame rates with lower readout noise levels, and making it the preferred choice for real-time experiments with very low neutron counts. For some applications an amorphous silicon flat panel detector is also suitable. At PSI the chosen camera is mounted in a camera box that gives adjustable focal distances, field of view (FOV) dimensions, and image pixel resolution. The standard PSI boxes allow an FOV of 15-300 $\mathrm{mm}$ and pixel sizes down to $6.5 \mu \mathrm{m}$.

When designing an experiment the key consideration when selecting the camera is its ability to resolve the details of interest with sufficient spatial and temporal resolution. The contrast between the phases in the sample also plays an important role. The contrast between phases occurs because each phase or component in the sample has a different attenuation coefficient. The number of captured neutrons determines how well this contrast can be resolved, with the difference between attenuation coefficients and the signalto-noise ratio determining detectability. Figure 1 shows the average number neutrons $(N)$ that can be detected for different exposure times and pixel sizes for a neutron flux of $10^{7}$ neutrons $\mathrm{cm}^{-2} \mathrm{~s}^{-1}$. The signal-to-noise ratio (SNR) is related to the neutron count at the detector by $\sqrt{N}$ since the noise has a Poisson distribution. The Poisson nature of the noise makes the SNR vary across the image with sample dimensions and composition. The scaling of the number of captured neutrons to the grayscale values presented in the images varies with the configuration of detection system, i.e., the scintillator-lens-camera combination used. 


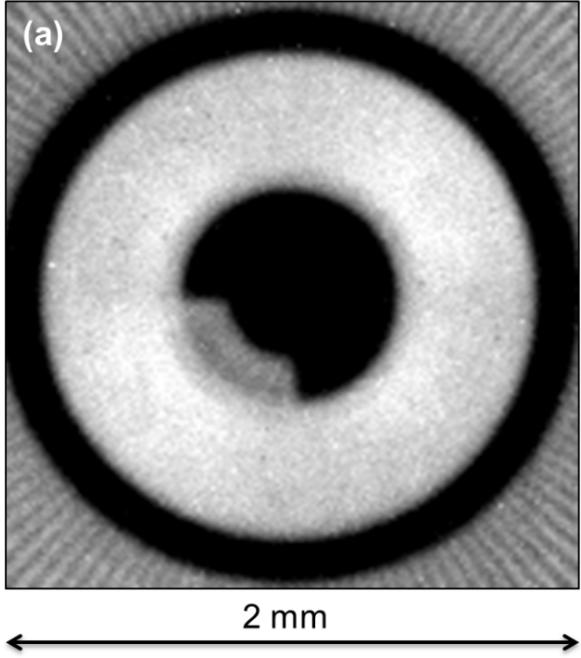

$13.5 \mu \mathrm{m}$ pixel size

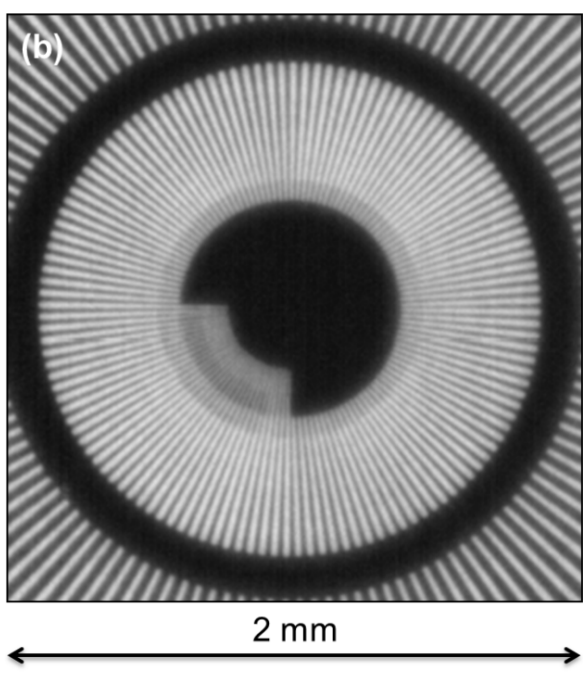

$1.5 \mu \mathrm{m}$ pixel size

Figure 2. Comparison of the neutron radiographs of the gadolinium Siemens star test object based on (a) standard high-resolution neutron instrument (Kaestner et al., 2011a) and (b) the Neutron Microscope prototype (Trtik et al., 2015).

\subsection{Neutron imaging with high spatial resolution}

Most high-resolution neutron imaging systems today operate with resolutions on the order of several tens of microns. This applies to camera-scintillator-based systems (Kaestner et al., 2011a; Williams et al., 2012) as well as pixel detectors (Jakubek et al., 2005; Tremsin et al., 2012). Higher resolution can be achieved using an arrangement were the scintillator is tilted to a shallow angle to the beam, and combined with a mirror (Boillat et al., 2008). This approach has the ability to increase the spatial resolution in one direction while the resolution in the other direction remains unchanged, producing images with rectangular pixels and thus anisotropic resolution. More recently, sub-ten micron resolution has been achieved using a pixel detector in combination with a centroiding technique to estimate the impact position of each detected neutron (Vavrik et al., 2014). In contrast, achromatic neutron microscope based on axisymmetric focussing mirrors for neutrons (so-called Wolter optics) have the potential to yield significant improvements in neutron flux and spatial resolution (Liu et al., 2013), but the current resolution of cold neutron Wolter optics is still limited to several tens of microns.

A first prototype of a microscope that magnifies the radiograph by a factor of 4.3 on a gadolinium oxysulfide $4 \mu \mathrm{m}$ thick $\left(\mathrm{Gd}_{2} \mathrm{O}_{2} \mathrm{~S}: \mathrm{Tb}\right)$ scintillator screen has been developed at PSI within the Neutron Microscope Project. This system is based on a magnifying lens for light and a low-noise camera of either CCD or SCMOS type. Using this configuration, pixel sizes down to $1.5 \mu \mathrm{m}$ can be obtained at a resolution of $7.6 \mu \mathrm{m}$ (Trtik et al., 2015). Radiographs of a Siemens star test object obtained using the so-called regular "micro-setup" and the new Neutron Microscope clearly demonstrate the im- provement in spatial resolution (Fig. 2), with the increased exposure times being the natural consequence of the smaller pixels of the Neutron Microscope images. The Neutron Microscope image required a $25 \mathrm{~min}$ exposure time, while the micro-setup image was acquired in $90 \mathrm{~s}$.

To shorten the acquisition times, and therefore allow further practical improvement of the spatial resolution, scintillator screens based on isotopically enriched gadolinium oxysulfide $\left({ }^{157} \mathrm{Gd}_{2} \mathrm{O}_{2} \mathrm{~S}: \mathrm{Tb}\right)$ have been developed $(\mathrm{Tr}-$ tik and Lehmann, 2015). When linked to tailor-made highnumerical-aperture magnifying optics, these scintillators can provide images with a spatial resolution of about $5 \mu \mathrm{m}(\mathrm{Tr}-$ tik and Lehmann, 2016). A more advanced version of the Neutron Microscope is currently being designed and implemented at PSI as a self-contained instrument that can, with a moderate effort, be used at different beamlines.

\subsubsection{High-resolution neutron imaging of drying in a porous medium}

Despite the relatively long acquisition times needed for imaging with the prototype Neutron Microscope, the instrument already allows observation of even slower processes in radiographic mode.

A sample of a model porous medium was used to demonstrate the spatiotemporal capabilities of the Neutron Microscope at the BOA beamline. The sample consists of a $\mathrm{SiO}_{2}$ capillary (of 1.25 and $1.0 \mathrm{~mm}$ inner and outer diameter respectively) filled with stainless steel spheres of about $1.0 \mathrm{~mm}$ in diameter. The sample was initially saturated with $\mathrm{H}_{2} \mathrm{O}$ and the water left evaporating freely under ambient conditions. The field of view was equal to approximately $3 \times 3 \mathrm{~mm}^{2}$, and the pixel size of the images was equal to $6 \mu \mathrm{m}$ pixel size. This 

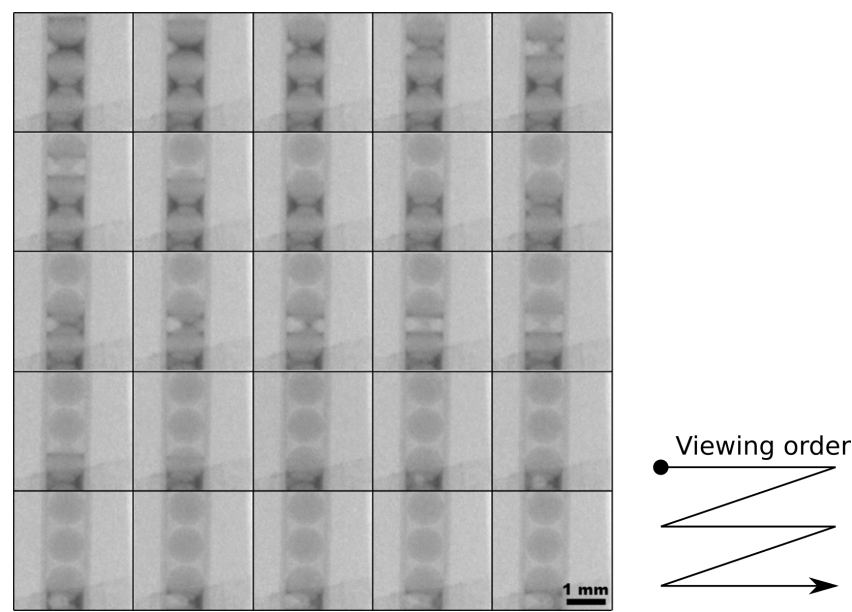

Figure 3. Time series of the evaporation of water from a model porous medium sample. The field of view is approximately $3 \times 3 \mathrm{~mm}^{2}$. The images were acquired with $6 \mu \mathrm{m}$ pixel size $(4 \times$ binning) using $90 \mathrm{~s}$ acquisition time. Only every 10th image is presented; therefore the time gap between the individual presented image is about $15 \mathrm{~min}$. The slightly darker trapezoidal object in the bottom right FOV is hydrogenous material within adhesive aluminum tape used for the sample fixation.

series was not acquired at highest possible spatial resolution. A $4 \times 4$ binning was used to increase the neutron dose per pixel. Individual projections' acquisition times were $90 \mathrm{~s}$.

Visualization of the time series (Fig. 3) demonstrates that processes occurring in porous media can be investigated with high spatial resolution in 2-D. The data revealed the heterogeneous removal of water (darkest gray/black) from the pore space between the capillary (near transparent) and the beads (mid-gray). It also shows that the water forms menisci at the solid-solid boundaries when air enters the system over the time span of more than $6 \mathrm{~h}$. The images are of sufficiently sharpness to allow quantitative measurements of the interfacial curvatures. The changes are clearly observable, even if the subsequent images are compared (every tenth image is presented in Fig. 3 for convenience reasons only).

Although the data presented are from a 2-D study, the quality of the images indicates that 3-D high-resolution neutron imaging of even slow processes could be performed when coupled to advanced reconstruction techniques (described later in Sect. 2.3).

\subsection{Time-lapse neutron CT}

The distribution of fluids in porous media can be considered as static under steady-state flow of a single fluid, or as dynamic under variable flow or composition. For the dynamic case, when capturing the effects of diffusion or flow processes over time is the objective of the experiment, timelapse or 4-D imaging is required. With neutron imaging, experimental approaches vary with the required contrast, spa- tial resolution, and the temporal development of the observed process. The main challenge during a dynamic CT experiment is to avoid artifacts caused by changes within the sample during imaging and the performance of the acquisition system. Reconstruction methods generally assume that the sample is invariant during the acquisition of all the projection data used for the reconstruction.

Changes to a sample during the acquisition of the data for a single 3-D image can be induced by vibrations and centrifugal forces imposed by the sample environment. However, in many experiments these effects can be neglected, and most artifacts relate to the acquisition rate. Motion artifacts can appear if the acquisition rate is low relative to the observed process. Sub-pixel displacement rates within the sample are sufficient to cause motion artifacts in the reconstructed data. The motion artifacts appear as tangential streaks emerging from the changing region, and poorly defined phase boundaries.

Increasing acquisition speeds would reduce motion artifacts, but can be counterproductive, as faster acquisition means lower SNR, and image quality will be substantially reduced leading to possibly coarser resolution. Hence, the challenge with 4-D CT experiments is to minimize the impact of motion artifacts while providing sufficient image contrast to allow observations of the process of interest in a sample that is unaffected by the acquisition procedure.

\subsubsection{High dynamic range and spatial resolution}

Detecting small changes in the fluid distribution with high spatial resolution requires longer exposure times to capture a sufficient neutron dose (number of detected neutrons per pixel) to resolve the attenuation contrast between phases. Exposure times on the order of several tens of seconds or even minutes per projection are not uncommon with neutron imaging. Thus, a typical duration for a neutron CT scan is several hours. Increasing the number of neutrons improves contrast and the signal-to-noise ratio, thereby improving postprocessing segmentation performance and reducing the errors in qualitative and quantitative analysis of the fluid distribution in the sample.

For most aqueous processes, the long exposure times mean that significant changes in the fluid distribution are likely over a single exposure, and that strong motion artifacts will appear in the reconstructed data. Two methods to reduce the impact of these artifacts use alternative acquisition sequences that have a time-averaging effect in the reconstructed data. The motion's artifact streaks are replaced by a smooth gradient across the extent of the dynamic region, which appears as edge unsharpness in the reconstructed data set, but does not affect static regions.

The first approach uses the repetition of several scans with equiangular increments each with a slight angular offset upon start. The second uses angular increments determined by the golden ratio (Köhler, 2004; Kaestner et al., 2011b). The lat- 
ter method is more flexible since the golden ratio generates an infinite series of angles that always spans the full angular range. Therefore, the acquisition can be stopped at any time, and reconstruction can be performed on any number of sequential projections from the data set. Scans that are too short do not satisfy the sampling theorem which can introduce sampling artifacts. The major advantage of these alternative acquisition sequences is that they can be decomposed into shorter sub-scans (i.e., each scan uses a subset of the total projections collected), therefore producing a time series of CT data from a single acquisition session. The time interval of each 3-D image (i.e., the number of projections used to reconstruct a single 3-D image) can be selected after the experiment. This means the exact start point and timescale of the phenomena in a specific sample do not have to be known ahead of the experiment. The timescale of the reconstructed data can be tuned during reconstruction by selecting an adequate subset of projections. Techniques for reconstructing this kind of data and an example with experiment data are described in Sect. 2.3 below.

\subsubsection{Fast acquisition}

When the process of interest is fast compared to the projection acquisition rate, the alternative acquisition schemes do not prevent motion artifacts. Under these conditions the projection (frame) acquisition rate must be increased at the cost of neutron dose. As a consequence, the dynamic range of the gray levels and SNR will be inferior in the reconstructed data. sCMOS cameras allow frame rates greater than 25 frames per second (making it possible to acquire an entire set of CT projection data in about $10 \mathrm{~s}$ ). Under such acquisition conditions, the sample table is continuously turning, while the camera acquires projections at a specified frame rate to avoid unnecessary time delay caused by discrete stepping. This so-called on-the-fly tomography was demonstrated by Zarebanadkouki et al. (2015), who acquired projections for tomogram in about $3 \mathrm{~min}$. However, turntable speed and the neutron dose are the limiting factors for on-the-fly experiments, especially at high spatial resolution.

The neutron dose per pixel can be improved by increasing the pixel size (Fig. 1), either by binning on the camera side or by increasing the focal distance of the lens; but this reduces the spatial resolution of the images. A low SNR in the experiment projection data increases the importance of high-quality reference images. Lower average neutron counts per pixel mean more reference images are needed to avoid contamination of the data by noise from both the open beam (flat/bright) and dark current (dark) images.

\subsubsection{Using on-the-fly tomography to study water uptake}

Rate and location of root water uptake by plants growing in soil is poorly understood due to the dynamic behavior of soil
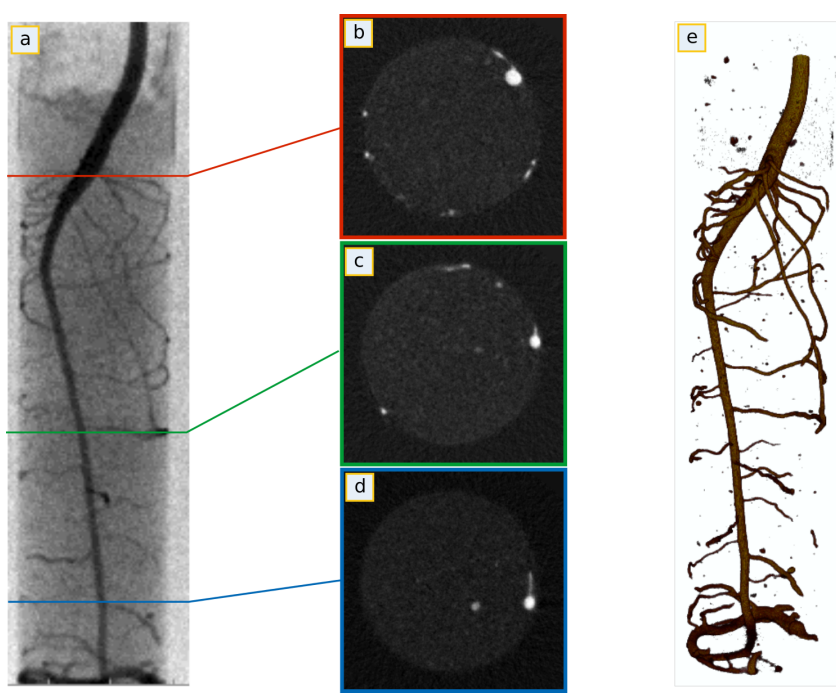

Figure 4. (a) Neutron radiography of a 2-week old lupine in a cylindrical container of sandy soil, $27 \mathrm{~mm}$ in diameter. (b, c, d) Horizontal slices of the reconstructed data at different depths indicated by the colored lines in the radiography. (e) 3-D rendering of the root system reconstructed using 181 projections

and roots of transpiring plants. In 2-D, high spatiotemporal resolution coupled to high sensitivity to the contrast between normal $\left(\mathrm{H}_{2} \mathrm{O}\right)$ and deuterated water $\left(\mathrm{D}_{2} \mathrm{O}\right)$ has allowed neutron radiography to successfully resolve average water uptake mechanism in a small Lupine plant (Zarebanadkouki et al., 2012, 2014). However, providing experimental data to validate the refine model and determining the relative contributions of the competing water transport pathways requires experimental quantification of the 3-D spatial distribution of $\mathrm{D}_{2} \mathrm{O}$ across the root tissue through time.

Performing these neutron tomography experiments was challenging because of the spatial and temporal resolution required; the process has been proven to take place within the course of several minutes depending on root thickness. Frame rates of $6 \mathrm{fps}$ were needed to remove motion artifacts (to keep the water displacements below one pixel over the entire 3-D data set) during on-the-fly tomography.

The cylindrical samples of sandy soil were $27 \mathrm{~mm}$ in diameter and $100 \mathrm{~mm}$ in length. $\mathrm{D}_{2} \mathrm{O}$ transport was imaged with a pixel size of $45 \mu \mathrm{m}$ using the Andor NEO sCMOS camera with an FOV of $55 \times 116 \mathrm{~mm}$ (corresponding to $1200 \times 2560$ pixels). The exposure time per projection was $167 \mathrm{~ms}$ (scan time of $30 \mathrm{~s}$ ). The $3-\mathrm{D}$ volumes were reconstructed from 181 projections uniformly distributed over 180 degrees. This represents a $6 \times$ increase in temporal resolution compared to Zarebanadkouki et al. (2015) and with no reduction in spatial resolution. A part of this speed increase is contributed to the higher neutron flux at ICON. The neutron dose here was about 100 neutrons pixel ${ }^{-1}$, which results in a lower SNR, but image quality is still acceptable for the investigation thanks to the high contrast between the different 


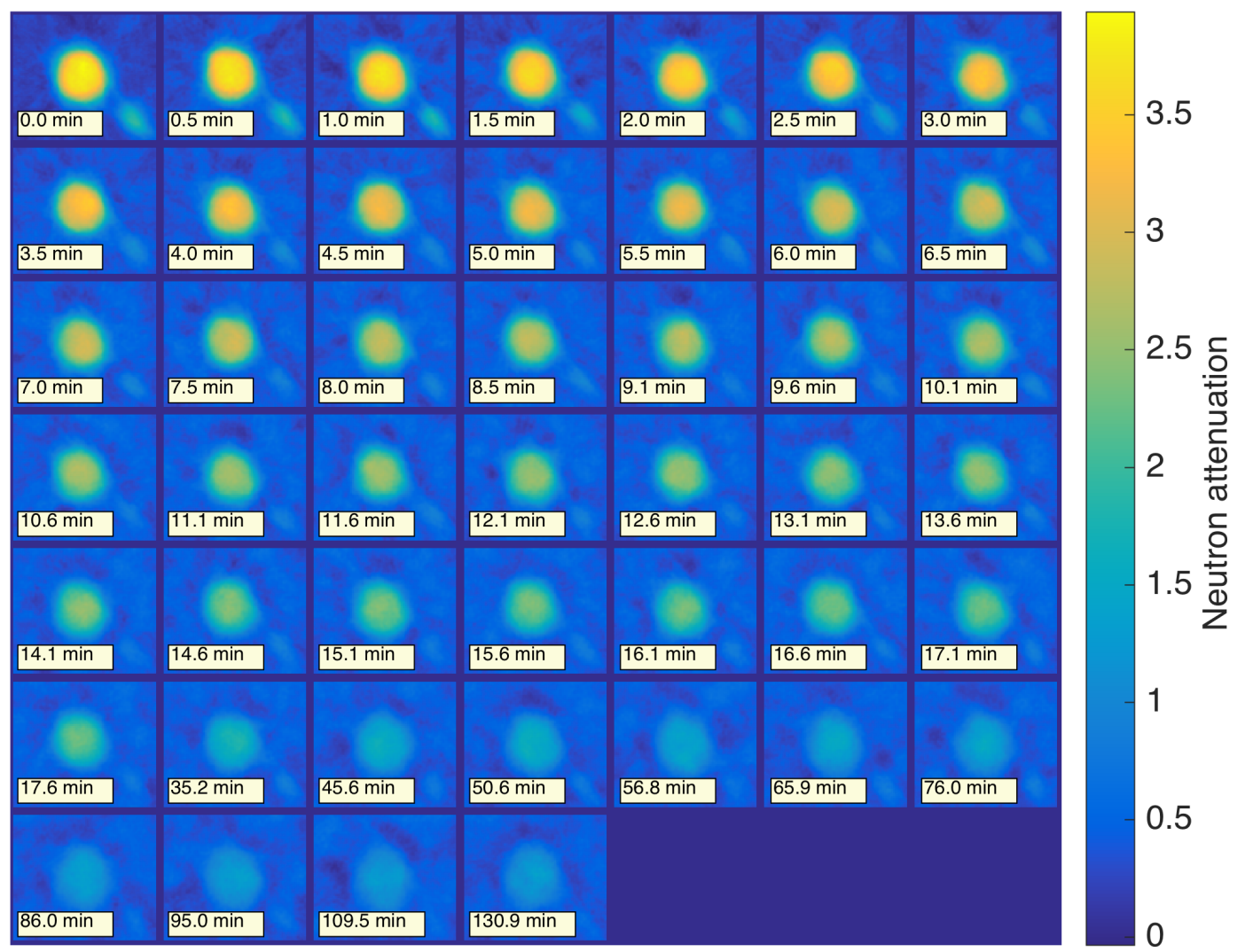

Figure 5. Time series neutron tomography of $\mathrm{D}_{2} \mathrm{O}$ transport into the root of a 2-week old lupine (nighttime). These images show the change in neutron attenuation across the root tissue at different times after $\mathrm{D}_{2} \mathrm{O}$ injection (time is indicated in minutes). As $\mathrm{D}_{2} \mathrm{O}$ enters the root tissue, $\mathrm{D}_{2} \mathrm{O}: \mathrm{H}_{2} \mathrm{O}$ increases and neutron attenuation decreases. $\mathrm{D}_{2} \mathrm{O}: \mathrm{H}_{2} \mathrm{O}$ is indicated by low attenuation (blue); low $\mathrm{D}_{2} \mathrm{O}: \mathrm{H}_{2} \mathrm{O}$ is indicated by high attenuation (yellow). The images show a $5 \times 5 \mathrm{~mm}$ region of one horizontal slice through the 3-D data at a depth of $50 \mathrm{~mm}$ from soil surface.

phases. The first 3-D data set represents the steady-state conditions before $\mathrm{D}_{2} \mathrm{O}$ was injected into the soil near the roots and its transport was simultaneously monitored using a time series neutron radiography (Figs. 4, 5).

The reconstructed data from the steady-state experiment (Fig. 4a) show that the image acquisition parameters provide sufficient contrast to enable segmentation of the roots from the soil.

Working at a single depth ( $50 \mathrm{~mm}$ from the soil surface) and tracking the $\mathrm{D}_{2} \mathrm{O}$ concentrations as a function of time (Fig. 5) shows the radial transport into the root. As $\mathrm{D}_{2} \mathrm{O}$ moves into the root, the neutron attenuation decreases with a sharp contrast between roots and their sounding soil. There is a distinct transition from $\mathrm{H}_{2} \mathrm{O}$ (yellow) to $\mathrm{D}_{2} \mathrm{O}$ ) (blue) over the first 5 min after $\mathrm{D}_{2} \mathrm{O}$ injection. This study proves that 4-D neutron (on-the-fly) tomography can capture the flow of water across the root tissue in 3-D with sufficiently high spatial and temporal resolution to verify root water uptake models. To this end, the transport of $\mathrm{D}_{2} \mathrm{O}$ across root tissue during day and night should be modeled by the diffusion-convection equation proposed in Zarebanadkouki et al. (2014). Note that for the case of dominant cell-to-cell transport, a sharp gradi- ent in concentration of $\mathrm{D}_{2} \mathrm{O}$ across the root tissue can be expected, while the gradient can be expected to be more gradual for the case of a dominant apoplastic pathway since $\mathrm{D}_{2} \mathrm{O}$ can quickly bypass the root tissue and reach the xylem. Data from 4-D imaging experiments showing the water distribution over time are urgently needed to verify models and to increase the understanding of the physical mechanisms controlling water, solute, and hormone transport in the roots.

\subsection{Towards new reconstruction methods}

Experimental projection data are usually reconstructed using the analytical filtered back projection (FBP) reconstruction algorithm (Buzug, 2008), which requires a large number of projections to perform well (i.e., to fulfill the sampling theorem). FBP is also sensitive to nonuniform angular sampling of the projection data. This can be a major problem when increasing acquisition speed for higher temporal resolution.

Undersampled projection data are best treated using iterative reconstruction techniques, as these can account for various inaccuracies required by the experimental method such as nonuniform sampling steps, low-intensity dynamics, and 

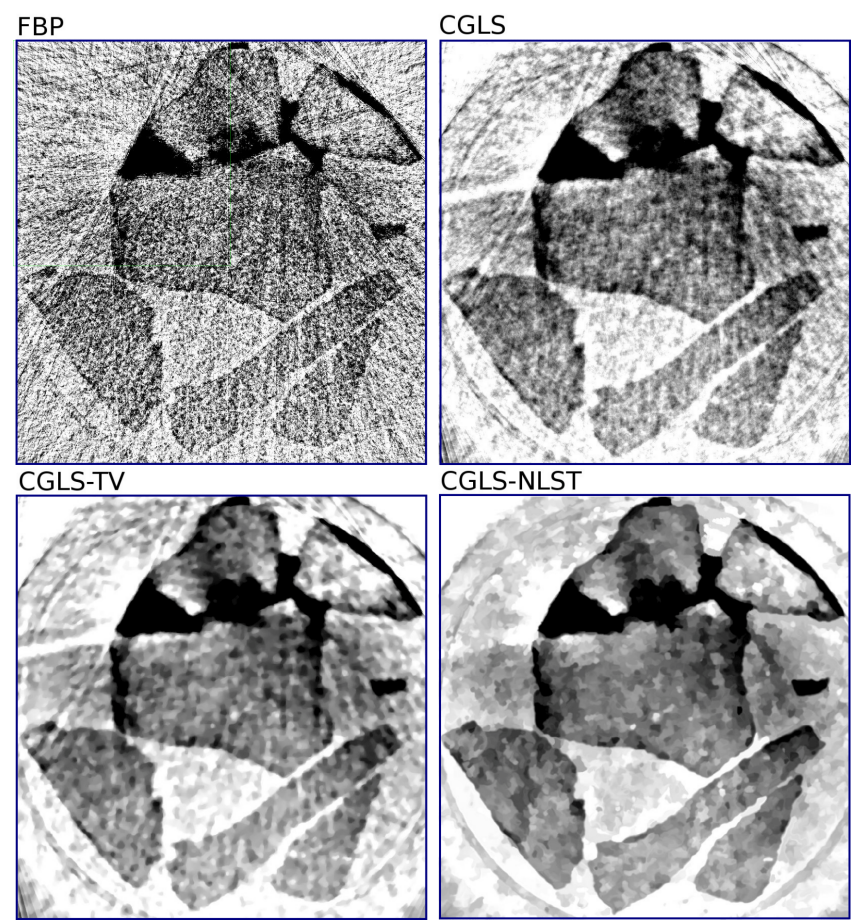

Figure 6. Reconstruction from 60 projections to show the difference iterative methods can make to resolving key spatial information in fluid flow studies. The images show an axial slice $(1000 \times 1000$ pixels) from one time frame of a 4-D experiment performed at ICON. The darkest areas depict the different times showing the progress of water entering the sample. The mid-gray regions are gravel particles which have approx. $10 \%$ internal porosity.

SNR in the measurements. These reconstruction techniques also allow the imposition of some regularity (e.g., smoothness) or data consistency (e.g., rejection of nonzero values) into the process to find the solution. The benefit hereby is to improve image quality, particularly for undersampled and under-exposed projection data.

Commonly, a priori information assumes some expected local intensity correlations, i.e., regions that remain unchanged throughout the experiment. In the fluid-flow problems of interest here, the initial stationary "dry" stage is an ideal "prior" reference for iterative reconstruction. Simultaneous iterative reconstruction (SIRT) and the conjugate gradient least square (CGLS) methods employed are now available (Van Eyndhoven et al., 2015; Kazantsev et al., 2015a). Here we summarize the findings that are of specific interest to porous media experiments. Figure 6 shows a $1000 \times 1000$ pixel slice reconstructed using the different methods to demonstrate the difference in performance using undersampled projection data (60 projections acquired using golden ratio protocols, see Sect. 2.2.1). This is $4 \%$ of the data needed to fulfill the Nyquist theorem; i.e., severely undersampled and strong streak artifacts are to be expected. The

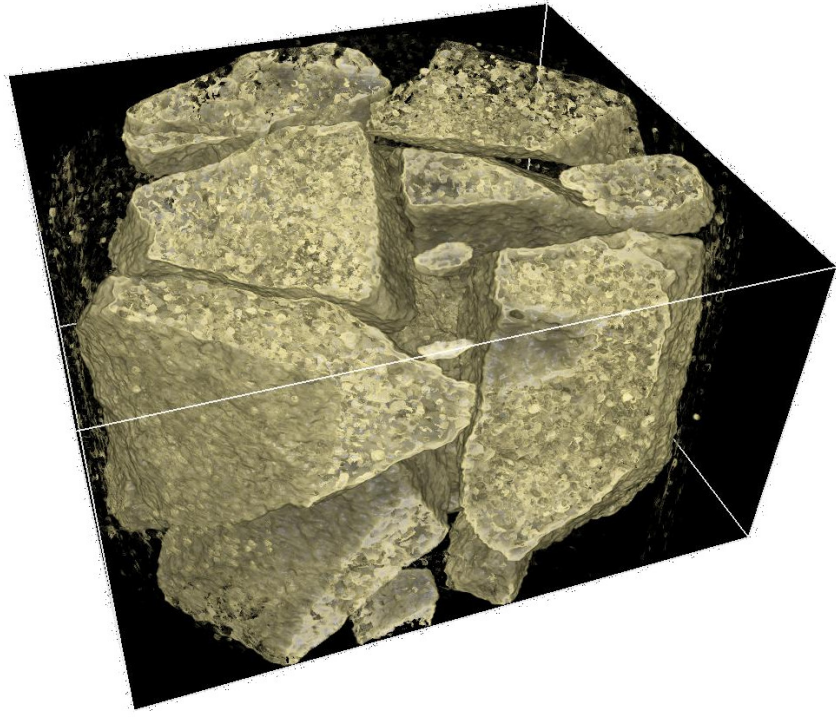

Figure 7. Rendered volume of the stationary "dry" sample (sandstone) prior to water ingress. Reconstruction from 150 projections using a CGLS-TV algorithm.

slices were reconstructed using FBP and three different iterative methods.

It can be seen that the CGLS-NLST (non-local spatiotemporal) method (Kazantsev et al., 2015a) gives significantly better resolution (sharper transitions between different phases) and better contrast separation between the phases. Sampling artifacts are much weaker and the SNR is higher in these data since the regularization is driven by the a priori information provided by a pre-reconstructed "dry" stationary initial image (Fig. 7) reconstructed from 150 projections using a CGLS-TV algorithm.

It is possible to reconstruct a time-lapse series showing the condition of the sample, using smaller subsets of projection data from one long sequence of time-evolving projection data, provided the data are acquired using the golden ratio method. The improved feature resolution provided by the CGLS-NLST reconstruction (Kazantsev et al., 2015a) allows us to quantify the liquid volumes in the sample through time at a minimum of $3 \times$ higher temporal resolution than possible with FBP, and at lower image noise (Fig. 8).

Another successful approach (Van Eyndhoven et al., 2015) for the reconstruction of undersampled time-lapse projection data uses the reconstructed stationary stage (i.e., extracting the rock phase) to define the stationary regions, and then iteratively refines the other phases (in this case air and water) by applying known intensity constraints (Kazantsev et al., 2015a, b; Van Eyndhoven et al., 2015).

The stationary region is redefined as the experiment progresses, so the reconstruction focusses on the dynamic regions only. This approach gives much better spatiotemporal resolution (data comparable to that of the CGLS-NLST method with fewer than 20 projections) than has been previ- 

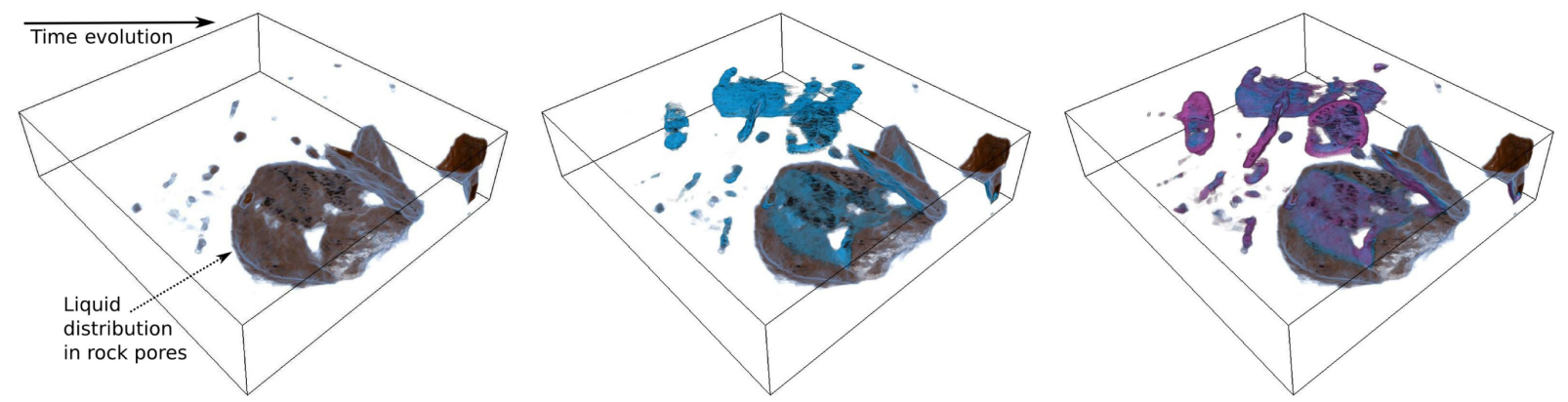

Figure 8. The fluid phase in the rock sample at three different times of the water ingress. Each volume is reconstructed from 60 projections using the CGLS-NLST method.
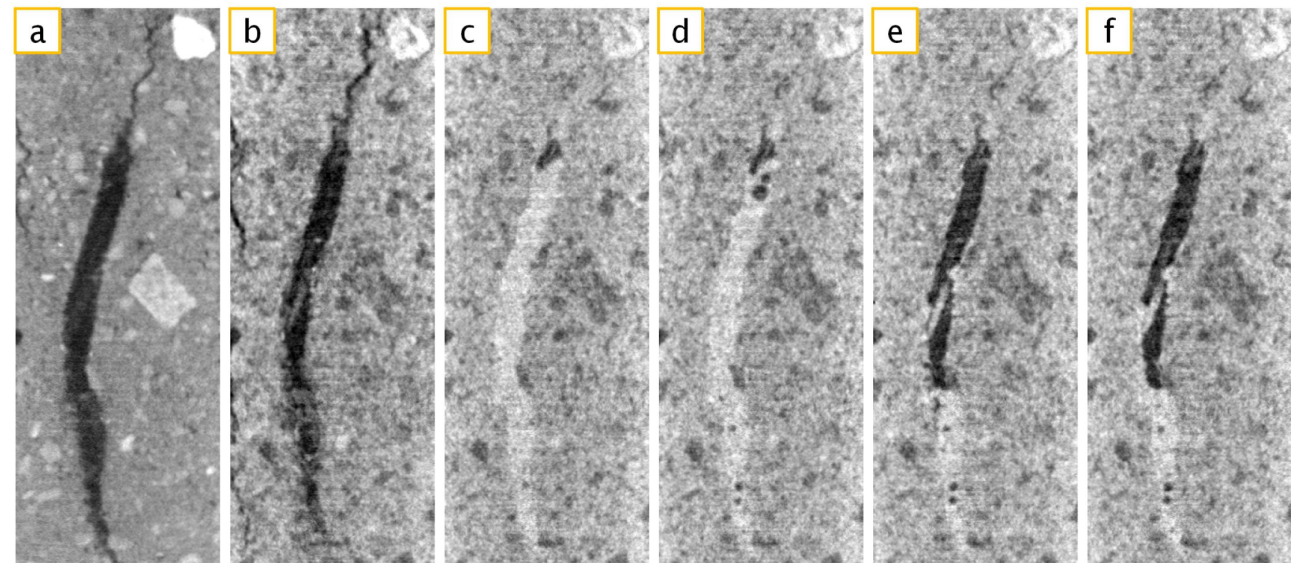

Figure 9. Cropped part $\left(22.1 \times 60.0 \mathrm{~mm}^{2}\right)$ of the vertical cross sections through the 3-D X-ray (a) and neutron data sets (b-c) of the intact soil sample taken into a cylinder (a diameter of $34 \mathrm{~mm}$, height of $87 \mathrm{~mm}$ ) during various stages of the recurrent ponded infiltration experiment: (a) and (b) before experiment when the macropore in the center of the image is filled with air, (c) during the infiltration when the macropore is entirely filled with water, (d) during continuing infiltration (e) after draining the sample, and (f) during second infiltration episode when the entrapped air formed in the macropore.

ously achieved (Kazantsev et al., 2014b, 2015a); however it is more parametrized and data-dependent. Current tests show that reconstructions from as few as 18 projections can be segmented.

\subsection{Bimodal imaging - combining neutron and X-ray imaging}

Every imaging modality has it strengths and weaknesses, and one method is rarely capable of providing all the information needed. Integrating data from a second imaging modality can provide important complementary information and reduce ambiguity. Combining neutron and X-ray CT is one example of bimodal imaging particularly relevant for investigations of flow in porous media. The attenuation coefficients of a particular phase will be significantly different in the two data sets, and therefore multivariate methods will provide better results than those using single data sources. In some cases the contrast is even reversed between the modalities, e.g., wa- ter which is low contrast for X-rays while it provides a high contrast for neutrons.

There are two typical uses for bimodal imaging: (1) the sample is static but it is difficult to identify relevant features due to small differences in contrast between the present materials and (2) samples with a dynamic component. In the latter case, one modality can be used to identify the static structures, while the other is used to capture the displacements caused by the observed process. For experiments with complex sample compositions, combining the two approaches to increase performance and data quality may be required.

Bimodal imaging using neutrons and X-rays is available to users at NEUTRA (Mannes et al., 2015; Vontobel et al., 2016) and will soon be operational at ICON (Kaestner et al., 2015). These neutron beamlines are also equipped with $X$ ray sources so both neutron and X-ray images can be performed in situ without moving the sample. X-ray and neutron images can be collected either simultaneously or interleaved (one modality after the other), removing the delays between scans that often occur when ex situ X-ray sources are used. 

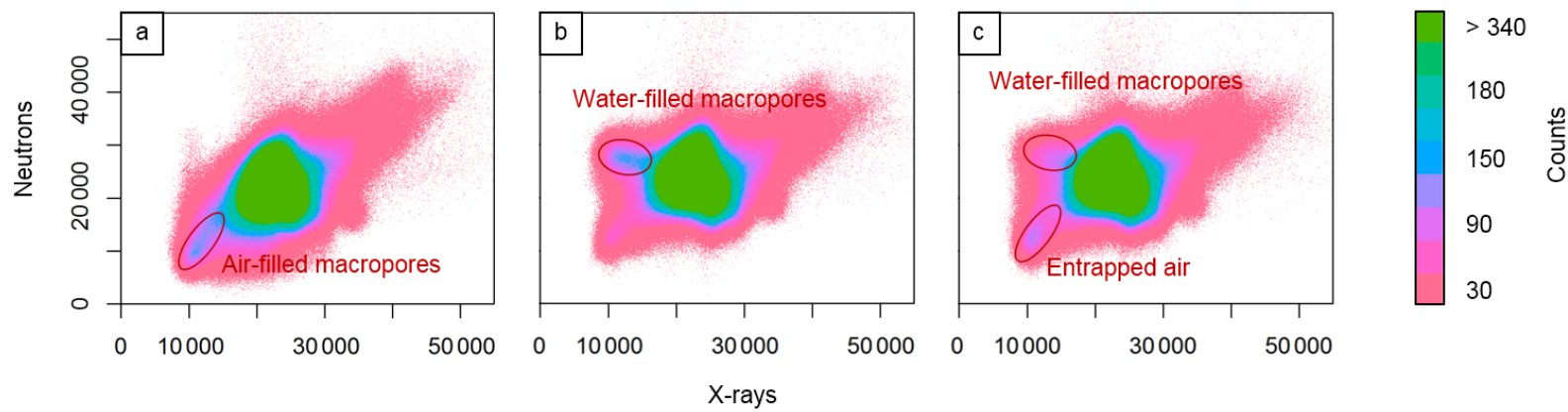

Figure 10. Bivariate histograms of cropped part of the X-ray and corresponding neutron tomograms, representing a soil sample with large macropores during various stages of recurrent ponding infiltration experiment. Histograms show (a) empty macropore before the infiltration was started, (b) filling of the macropores during the first infiltration, and (c) partial filling of the macropores during the second infiltration.
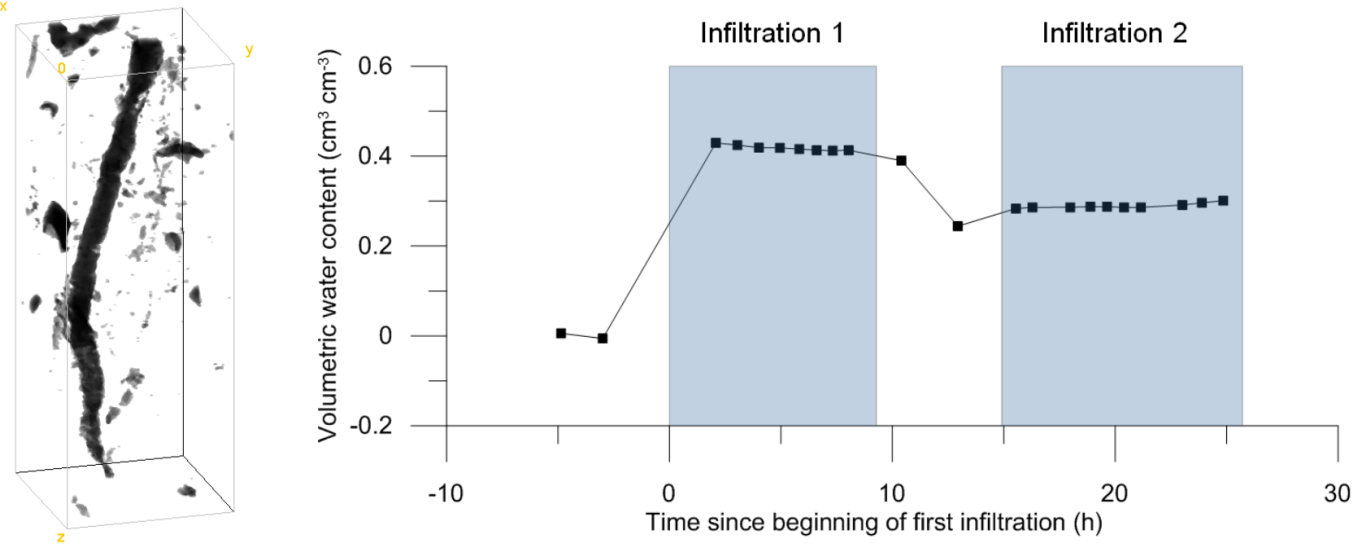

Figure 11. Macropore system that represents $1.97 \%$ of the sample volume segmented from X-ray tomogram (left). Time sequence of water contents in the macropore system derived from series of 22 tomograms (right). The blue columns indicate the time of two ponding infiltration episodes.

One key advantage of using the same sample manipulation environment is the reduced registration required to align the image data from the modalities onto a common coordinate system. This is in particular true for the installation at NEUTRA where the X-ray and neutron beams have a very similar geometry, and makes pixel-wise comparison possible. At ICON, an alternative approach has been taken, and the divergent X-ray source and beamline mounted perpendicular to the neutron beam direction. This installation further reduces the time between imaging using each modality, and makes simultaneous imaging using both modalities possible. The only need to drive ex situ X-ray imaging in this case is the potential for higher spatial resolutions that can be achieved elsewhere.

There are several strategies to analyze bivariate data. The work flow usually starts with a registration step to align the data sets onto a common coordinate system. This guarantees that pixel-/voxel-wise analysis of the two data sets is possible. Approaches that start data fusion during the reconstruction stage first register the projection data Kazantsev et al. (2014a). Other approaches reconstruct the data inde- pendently and then fuse at a later point in time. Development of these strategies is ongoing and the choice of data fusion strategy will depend both on the sample type and the information to be extracted from the experiment.

\subsubsection{Bimodal imaging to study preferential water flow in soil}

As an example of combined X-ray and neutron tomography imaging, we present a study of water and air behavior in soil. The infiltration of water and air trapping was studied on intact samples of coarse sandy loam soil from a mountainous site (Cambisol series, Korkusova Hut, Sumava Mountains, Czech Republic) from a depth of $40 \mathrm{~cm}$ below the surface. Preferential and localized water flow often occurs in this soil (Dohnal et al., 2012), with the majority of the water utilizing the small fraction of the soil volume characterized by soil macropores, fractures, and high-porosity regions, which results in uneven weathering. The preferential flow can be numerically simulated by dual-continuum models (e.g., Gerke et al., 2007). However, current models 
do not account for trapped air that can efficiently obstruct the macropores and significantly reduce soil permeability for water (Snehota et al., 2015); thus these model predictions fail.

The experiment performed at the NEUTRA beamline consisted of two infiltration episodes during which a layer of $\mathrm{D}_{2} \mathrm{O}: \mathrm{H}_{2} \mathrm{O}$ mixture (in the approx. mass ratio of $95: 5$ ) was maintained on the sample surface (a condition known as ponding), while water was left to drain the sample freely through the bottom by gravity. Flooding of the sample surface and maintaining the water level was controlled remotely. One X-ray and two neutron 3-D tomography data sets (tomograms) were acquired in the initial state before the first infiltration was begun. Another 20 neutron tomograms were acquired during the following $25 \mathrm{~h}$ of the experiment (see subset of the data in Fig. 9).

The neutron and X-ray tomograms were reconstructed by the MuhRec3 code (Kaestner, 2011) using 201 projections over $180^{\circ}$. The detector used for neutron imaging was a $\mathrm{LiF} / \mathrm{ZnS}$ scintillator screen, $100 \mu \mathrm{m}$ thick, photographed by a CCD camera (Andor iKON L BW936). The exposure time for each projection was $8 \mathrm{~s}$ and the acquisition time for one tomogram was approx. $50 \mathrm{~min}$. The nominal pixel size was $0.10 \times 0.10 \mathrm{~mm}^{2}$.

Fine co-registration of the X-ray and neutron tomograms was done by searching for the minimum of sum of differences while rotating and translating the X-ray tomogram as a rigid body. Bivariate histograms shown in Fig. 10 helped identify a threshold of 14500 a.u. for subsequent segmentation of the macropores from the X-ray data.

The segmented $\mathrm{X}$-ray tomogram was then used as a binary mask (see Fig. 11, left) before the evolving water volume in the macropores was determined from the neutron tomograms. The volume of water and average water content in the macropore system was calculated by subtracting the data from the dry sample and dividing the grayscale values by the attenuation coefficient of water (Fig. 11, right). The data show a reduction in the water content prior to and during the second infiltration, which results from air trapping in some of the pores. The data are being used to further develop the follow-up model by Fucik et al. (2010) that is based on the two-phase flow approach.

\section{Conclusions}

We have shown, using state-of-the-art approaches, that neutron imaging beamlines are a vital tool for observing processes in porous media. Routine methods are now approaching, or exceeding the spatial and temporal scales that can be achieved with lab-based X-ray sources. However, it is this capability coupled to the high sensitivity to hydrogen that makes neutron imaging such an ideal probe for highresolution experiments (2-D or 3-D over time) into the spatiotemporal distribution of fluids in porous media. PSI in- strument options achieve spatial resolutions on the order of a $1-50 \mu \mathrm{m}$ and 3-D image acquisition rates $1-7 \mathrm{CT}$ volumes $\min ^{-1}$; yet, often this was achieved at the cost of poorer image quality (consequence of lower signal-to-noise ratio). The combination of experiment equipment and numerical methods summarized here marks a major step forwards in this regard.

While it is important that the data can be acquired, the techniques shown here aim to increase the information that can be extracted from the data as well as increase the data acquisition rate. The neutron imaging community is currently focussed on developing improved methods for multidimensional and multimodal data analysis under low SNR conditions through close collaboration between the user community, instrument scientists, and algorithm developers. For experiments that produce data with intrinsic time structure, it is often beneficial to perform the analysis in 4-D instead of processing the data as a sequence of individual 3-D images. Ideally, the subsequent post-processing quantitative analyses will also involve the time structure to yield more accurate analyses.

Already, thanks to recent technological improvements, neutron imaging is a good choice to observe and quantify transport processes in porous media at different scales in time and space, regardless of whether the pore space can be resolved or not.

Acknowledgements. For the experiments performed at NEUTRA, we kindly acknowledge the instrument support by Jan Hovind, Martina Sobotkova, and Vladka Jelinkova. We would also like to acknowledge the support and funding from COST action MP1207, the Czech Science Foundation (project no. 14-03691S), as well as the European Union's Seventh Framework Programme for Research and Technological Development under the NMI3-II grant no. 283883, SINQ 20110581.

Edited by: H. Steeb

Reviewed by: F. Fusseis and R. Jänicke

\section{References}

Anderson, I., McGreavy, R., and Bilheux, H. (Eds.): Neutron Imaging and Applications, Springer Verlag, 2009.

Boillat, P., Kramer, D., Seyfang, B., Frei, G., Lehmann, E., Scherer, G., Wokaun, A., Ichikawa, Y., Tasaki, Y., and Shinohara, K.: In situ observation of the water distribution across a PEFC using high resolution neutron radiography, Electrochem. Commun., 10, 546-550, 2008.

Buzug, T.: Introduction to Computed Tomography: From photon statistics to modern cone-beam CT, Springer, 2008.

Carminati, A., Kaestner, A., Ippisch, O., Koliji, A., Lehmann, P., Hassanein, R., Vontobel, P., Lehmann, E., Laloui, L., Vulliet, L., and Flühler, H.: Water flow between soil aggregates, Transport Porous Med., 68, 219-236, 2007. 
Cnudde, V. and Boone, M.: High-resolution X-ray computed tomography in geosciences: A review of the current technology and applications, Earth-Sci. Rev., 123, 1-17, 2013.

Dobson, K. J., Coban, S. B., McDonald, S. A., Walsh, J. N., Atwood, R. C., and Withers, P. J.: 4-D imaging of sub-second dynamics in pore-scale processes using real-time synchrotron $\mathrm{X}$ ray tomography, Solid Earth, 7, 1059-1073, doi:10.5194/se-71059-2016, 2016.

Dohnal, M., Vogel, T., Sanda, M., and Jelinkova, V.: Uncertainty analysis of a dual-continuum model used to simulate subsurface hillslope runoff involving oxygen-18 as natural tracer, J. Hydrol. Hydromech., 60, 194-205, 2012.

Fucik, R., Sakaki, J. M. T., Benes, M., and Illangasekare, T.: Significance of dynamic effect in capillarity during drainage experiments in layered porous media, Vadose Zone J., 9, 697-708, 2010.

Gerke, H., Dusek, J., Vogel, T., and Kohne, J.: Two-dimensional dual-permeability analyses of a bromide tracer experiment on a tile-drained field, Vadose Zone J., 6, 651-667, 2007.

Hassanein, R.: Correction methods for the quantitative evaluation of thermal neutron tomography, Phd. thesis eth no. 16809, Swiss Federal Institute of Technology, doi:10.3929/ethz-a-005273682, 2006.

Jakubek, J., Holy, T., Lehmann, E., Pospisil, S., Uher, J., Vacikc, J., and Vavrika, D.: Spatial resolution of Medipixnext term-2 device as neutron pixel detector, Nucl. Instrum. Meth. A, 546, 164-169, 2005.

Kaestner, A.: MuhRec - a new tomography reconstructor, Nucl. Instrum. Meth. A, 651, 156-160, 2011.

Kaestner, A., Hartmann, S., Kuehne, G., Frei, G., Gruenzweig, C., Josic, L., Schmid, F., and Lehmann, E.: The ICON beamline - A facility for cold neutron imaging at SINQ, Nucl. Instrum. Meth. A, 659, 387-393, 2011.

Kaestner, A. P., Münch, B., Trtik, P., and Butler, L. G.: Spatiotemporal computed tomography of dynamic processes, Opt. Eng., 50, 123201, doi:10.1117/1.3660298, 2011.

Kaestner, A., Morgano, M., Hovind, J., and Lehmann, E.: Bimodal Imaging Using Neutrons and X-rays, in: Proceedings of the International Symposium on Digital Industrial Radiology and Computed Tomography, http://www.ndt.net/events/DIR2015/Paper/ 58_Kaestner.pdf (last access: 19 August 2016), 2015.

Kardjilov, N., Manke, I., Hilger, A., Strobl, M., and Banhart, J.: Neutron imaging in materials science, Mater. Today, 14, 248256, 2011

Kazantsev, D., Ourselin, S., Hutton, B. F., Dobson, K. J., Kaestner, A. P., Lionheart, W. R. B., Withers, P. J., Lee, P. D., and Arridge, S. R.: A novel technique to incorporate structural prior information into multi-modal tomographic reconstruction, Inverse Probl., 30, 065004, doi:10.1088/0266-5611/30/6/065004, 2014a.

Kazantsev, D., Thompson, W., Dobson, K. J., Kaestner, A., Lionheart, W., Withers, P., and Lee, P.: Simultaneous 4D-CT reconstruction using higher order spatial regularization and edge enhancement based on global information in temporal domain, Inverse Probl. Imag., 2014b.

Kazantsev, D., Eyndhoven, G. V., Lionheart, W., Withers, P., Dobson, K., McDonald, S., Atwood, R., and Lee, P.: Employing temporal self-similarity across the entire time domain in computed tomography reconstruction, P. T. R. Soc. London A, 373, 1-14, 2015a.
Kazantsev, D., Thompson, W. M., Lionheart, W. R. B., Eyndhoven, G. V., Kaestner, A. P., Dobson, K. J., Withers, P. J., and Lee, P. D.: 4D-CT reconstruction with unified spatial-temporal patch-based regularization, Inverse Probl. Imag., 9, 447-467, doi:10.3934/ipi.2015.9.447, 2015b.

Köhler, T.: A Projection Access Scheme for Iterative Reconstruction Based on the Golden Section, in: Nuclear Science Symposium Conference Record, IEEE, vol. 6, 3961-3965, 2004.

Krane, K.: Introductory nuclear physics, John Wiley \& Sons, 845 pp., 1988.

Lal, S., Poulikakos, L., Gilani, M. S., Jerjen, I., Vontobel, P., Partl, M., Carmeliet, J., and Derome, D.: Investigation of Water Uptake in Porous Asphalt Concrete Using Neutron Radiography, Transport Porous Med., 105, 431-450, 2014.

Lehmann, E. and Kaestner, A.: 3D neutron imaging, Encyclopedia of Analytical Chemistry, a9123, doi:10.1002/9780470027318.a9123, 2009.

Lehmann, E. H., Vontobel, P., and Wiezel, L.: Properties of the radiography facility NEUTRA at SINQ and it's potential for use as European reference facility, Nondestruct. Test. Eva., 16, 191$202,2001$.

Liu, D., Hussey, D., Gubarev, M. V., Ramsey, B. D., Jacobson, D., Arif, M., Moncton, D. E., and Khaykovich, B.: Demonstration of achromatic cold-neutron microscope utilizing axisymmetric focusing mirrors, Appl. Phys. Lett., 102, 183508 , doi:10.1063/1.4804178, 2013.

Mannes, D., Schmid, F., Frey, J., Schmidt-Ott, K., and Lehmann, E.: Combined Neutron and X-ray imaging for non-invasive investigations of cultural heritage objects, Physics Procedia, 69, 653-660, 2015.

Morgano, M., Peetermans, S., Lehmann, E., Panzner, T., and Filges, U.: Neutron imaging options at the BOA beamline at Paul Scherrer Institut, Nucl. Instrum. Meth. A, 754, 46-56, 2014.

Robinson, B., Moradi, A., Schulin, R., Lehmann, E., and Kaestner, A.: Neutron Radiography for the Analysis of Plant-Soil Interactions, Encyclo, a9023, doi:10.1002/9780470027318.a9023, 2008.

Rudolph, N., Esser, H. G., Carminati, A., Moradi, A. B., Hilger, A., Kardjilov, N., Nagl, S., and Oswald, S. E.: Dynamic oxygen mapping in the root zone by fluorescence dye imaging combined with neutron radiography, J. Soil. Sediment., 12, 63-74, 2012.

Schaap, J., Lehmannn, P., Kaestner, A., Vontobel, P., Hassanein, R., Frei, G., de Rooij, G., Lehmann, E., and Flühler, H.: Measuring the effect of structural connectivity on the water dynamics in heterogeneous porous media using speedy neutron tomography, Adv. Water Resour., 31, 1233-1241, 2008.

Sears, V.: Neutron scattering lengths and cross sections, Neutron News, 3, 26-37, doi:10.1080/10448639208218770, 1992.

Snehota, M., Jelinkova, V., Sobotkova, M., Sacha, J., Vontobel, P., and Hovind, J.: Water and entrapped air redistribution in heterogeneous sand sample: Quantitative neutron imaging of the process, Water Resour. Res., 51, 1359-1371, 2015.

Tremsin, A., McPhate, J., Vallerga, J., Siegmund, O., Feller, W., Lehmann, E., Kaestner, A., Boillat, P., Panzner, T., and Filges, U.: Neutron radiography with sub- $15 \mu \mathrm{m}$ resolution through event centroiding, Nucl. Instrum. Meth. A, 688, 32-40, 2012.

Trtik, P. and Lehmann, E.: Isotopically-enriched gadolinium-157 oxysulfide scintillator screens for the high-resolution neutron imaging, Nucl. Instrum. Meth. A, 788, 67-70, 2015. 
Trtik, P. and Lehmann, E.: Progress in High-resolution Neutron Imaging at the Paul Scherrer Institut - The Neutron Microscope Project, J. Phys. Conf. Ser., in press, 2016.

Trtik, P., Hovind, J., Grünzweig, C., Bollhalder, A., Thominet, V., David, C., Kaestner, A., and Lehmann, E. H.: Improving the spatial resolution of neutron imaging at Paul Scherrer Institut - The Neutron Microscope Project, Physics Procedia, 69, 169176, 2015.

Van Eyndhoven, G., Batenburg, K., Kazantsev, D., Van Nieuwenhove, V., Lee, P., Dobson, K., and Sijbers, J.: An Iterative CT Reconstruction Algorithm for Fast Fluid Flow Imaging, IEEE T. Image Process., 24, 4446-4458, 2015.

Vavrik, D., Jakubek, J., Kaestner, A., Krejci, F., Turecek, D., and Zemlicka, J.: Modular pixelated detector system for neutron imaging with micrometric resolution, in: 10th World conference for neutron radiography - Book of abstracts, 2014.

Vontobel, P., Mannes, D., Kaestner, A., Schmid, F., and Lehmann, E. H.: The X-ray option at the NEUTRA imaging beamline of the spallation neutron source SINQ, Nuclear Instruments \& Methods in Physics Research, Section A: Accelerators, Spectrometers, Detectors, and Associated Equipment, in review, 2016.

Wildenschild, D. and Sheppard, A. P.: X-ray imaging and analysis techniques for quantifying pore-scale structure and processes in subsurface porous medium systems, Adv. Water Resour., 51, 217-246, 2013.
Williams, S., Hilger, A., Kardjilov, N., Manke, I., Strobl, M., Douissard, P., Martin, T., Riesemeier, H., and Banhart, J.: Detection system for microimaging with neutrons, J. Instrum., 7, P02014, doi:10.1088/1748-0221/7/02/P02014, 2012.

Zarebanadkouki, M., Kim, Y. X., Moradi, A. B., Vogel, H. J., Kaestner, A., and Carminati, A.: Quantification and Modeling of Local Root Water Uptake Using Neutron Radiography and Deuterated Water, Vadose Zone J., 11, 3, doi:10.2136/vzj2011.0196, 2012.

Zarebanadkouki, M., Kroener, E., Kaestner, A., and Carminati, A.: Visualization of root water uptake: quantification of deuterated water transport in roots using neutron radiography and numerical modeling, Plant Physiol., 166, 487-499, 2014.

Zarebanadkouki, M., Carminati, A., Kaestner, A., Mannes, D., Morgano, M., Peetermans, S., Lehmann, E., and Trtik, P.: Onthe-fly neutron tomography of water transport into lupine roots, Physics Procedia, 69, 292-298, 2015.

Zhang, P., Wittmann, F., Zhao, T., and Lehmann, E.: Neutron imaging of water penetration into cracked steel reinforced concrete, Physica B, 405, 1866-1871, 2010. 